\title{
CAPÍTULO 91
}

\section{TECNOLOGIA EDUCATIVA EM SAÚDE COMO UMA FERRAMENTA DE PROMOVER O BEM ESTAR EM TEMPOS DE PANDEMIA DA COVID-19: REVISÃO INTEGRATIVA}

DOI 10.4322/978-65-995353-2-1.c91

\author{
$\underline{\text { Antonia Mylene Sousa Almeida }}{ }^{1}$, Jhonnata Bezerra Araújo Melo ${ }^{2}$, Lorena Stephany Lopes
} Fernandes ${ }^{3}$, Victória de Souza Nery ${ }^{4}$, Isis Passos Ferreira ${ }^{5}$, Isabelly Raiane Silva dos Santos ${ }^{6}$, Jônatas Lucas Marcelino da Silva ${ }^{7}$, Mylena Sousa Almeida ${ }^{8}$, Regisson Cauann Teixeira Barbosa $^{9}$, Geísa de Morais Santana ${ }^{10}$

\author{
${ }^{1}$ Faculdade de Educação São Francisco, (mylenesousa123@hotmail.com) \\ ${ }^{2}$ Faculdade de Educação São Francisco, (jhonnatamelo13@gmail.com) \\ ${ }^{3}$ Centro Universitário Uninovafapi, (lorenastephanny_12@hotmail.com) \\ ${ }^{4}$ Faculdade São Francisco da Paraíba (victorianery2001@ @otmail.com) \\ ${ }^{5}$ Centro Universitário Fametro (isispassosferreira@ gmail.com) \\ ${ }^{6}$ Universidade Federal do Pará (isabelly.santos@ifpa.edu.br) \\ ${ }^{7}$ Universidade Federal de Pernambuco (jônatas.lucas@ufpe.br) \\ ${ }^{8}$ Faculdade de Educação São Francisco, (smylena191@gmail.com) \\ ${ }^{9}$ Centro Universitário Santo Agostinho (cauannt@gmail.com) \\ ${ }^{10}$ Universidade Estadual do Piauí, (geisasantana97@gmail.com)
}

\section{Resumo}

O presente estudo têm-se como objetivo identificar os diferentes tipos de educação em saúde através das tecnologias em tempos de pandemia, como também discutir sobre a importância das tecnologias para disseminar informações em meio a pandemia da COVID-19. Trata-se de uma revisão integrativa da literatura, no qual o levantamento se deu através das bases de dados: Base de Dados de Enfermagem (BDENF via BVS), Medical Literature Analysis and Retrieval System Online (MEDLINE via PUBMED) e Literatura Latino-americana e do Caribe em Ciências da Saúde (LILACS via BVS). Para esse trabalho, foram considerados como critérios de inclusão os artigos originais disponíveis por meio eletrônico em português e/ou em inglês e os que estão disponíveis entre o ano de 2020 e 2021, onde correspondem ao tempo de pandemia. Os critério de exclusão, são artigos que não tratam da temática proposta, artigos duplicados, teses, monografias, dissertações, revisão, livros e que apresenta texto incompleto. A partir disso, o método de busca foi desenvolvido por meio da PICO no qual Population: coronavírus; Intervention: tecnologias da informação; 
Comparison: não há e Outcomes: educação em saúde. A seleção dos descritores controlados e não controlados foram através dos DeCS, e do MeSH. Foram cruzados através do operador booleano "AND" para busca simultânea dos assuntos. Dentre os principais resultados, as tecnologias de informação contribuem para o repasse dos conhecimentos e da conversação beneficiando a população, além disso o uso das tecnologias é a forma mais eficaz nesse período, pois proporciona um maior alcance das orientações, podendo chegar à maioria da população. Devido ao fato da COVID-19 ser uma doença de alta transmissão, torna-se irrealizável as ações comunitárias em saúde. Diante do exposto, o uso das tecnologias em tempos de pandemia da COVID-19 é urgente e essencial, visto que é uma estratégia inovadora e eficaz para as ações educativas.

Palavras-chave: Coronavírus; Tecnologia da Informação; Educação em saúde.

Área Temática: Ciências da Saúde

E-mail do autor principal: mylenesousa123@ hotmail.com

\section{INTRODUÇÃO}

Segundo Pimentel et al. (2020), os coronavírus constituem uma grande família de vírus e sua nomeação se dá devido às espículas na superficie, que lembram uma coroa. Existem seis espécies desse vírus que são capazes de adoecer a espécie humana, o SARS-COV-2 corresponde ao sétimo coronavírus que causa a doença COVID-19.

A COVID-19 foi identificada na cidade da China em Wuhan no dia 31 de dezembro de 2019 e desde então vem trazendo desafios em todo o mundo (GRANJEIRO et al. 2020). As pessoas com essa doença podem apresentar sintomas como tosse, dores na garganta, dificuldade de respirar, febre e outras manifestações clínicas, como também podem ser assintomáticos (CAVALCANTE et al. 2020). O vírus da COVID-19 é de fácil transmissão através de gotículas pelas vias respiratórias, como também pelo contato físico (SOARES et al. 2020).

De acordo com Granjeiro et al. (2020), o Ministério da Saúde divulgou recomendações para interromper a disseminação do vírus, no qual uma das principais delas foi o isolamento social afim de separar pessoas sintomáticas ou assintomáticas de maneira a conter a transmissão da doença. Diante desse cenário, onde a maior porcentagem da população se encontra em suas residências, vêse a importância da tecnologia para realizar as ações na produção da saúde. Dentre as tecnologias, pode-se relatar sobre as tecnologias educacionais que tem a finalidade de facilitar o processo de educação em saúde da população com a transmissão de conhecimentos sobre a doença, tratamento e o autocuidado (MANIVA et al. 2017).

Portanto, essa pesquisa torna-se relevante devido a importância de abordar de forma especifica sobre a educação em saúde em tempos de pandemia da COVID-19, tendo em vista o cenário atual onde a população encontra-se em isolamento e necessitada de informações para a promoção, 
prevenção e recuperação da doença. A pergunta norteadora para essa pesquisa, foi a seguinte: de que forma as tecnologias da informação podem promover educação em saúde na pandemia da coronavírus?

\section{OBJETIVOS}

Identificar os diferentes tipos de educação em saúde através das tecnologias em tempos de pandemia, como também discutir sobre a importância das tecnologias para disseminar informações em meio a pandemia da COVID-19.

\section{MÉTODO}

Esse estudo é baseado na revisão integrativa da literatura, na qual a coleta de dados deu início no período de abril e finalizou em maio de 2021. De acordo com Souza, Silva e Carvalho (2010), a revisão integrativa é um instrumento da prática baseada em evidências, bem como um tipo de método que auxilia na produção de informações e na aplicação dos resultados obtidos.

O método de estudo abordado nesse trabalho apresenta informações sobre um tema específico, visto que tem a finalidade de reconhecer, avaliar e produzir resultados de estudos. Esse método consiste em seis fases para a preparação da revisão, são elas: criação da pergunta que irá nortear o trabalho; busca de dados; coleta de dados; análise dos conteúdos selecionados; discussão dos resultados; apresentação da revisão (SOUZA; SILVA; CARVALHO, 2010).

Santos, Pimenta e Nobre (2007) afirmam que a estratégia PICO é fundamental para a construção da pergunta norteadora e para a busca nas bases de dados. PICO retrata um acrômio para P: paciente; I: intervenção; C: comparação; O: outcomes (desfecho). Para a realização da construção da pergunta norteadora, considerou-se: P: coronavírus; Intervenção: tecnologias da informação; Comparação: não existe; Outcomes: educação em saúde. A pergunta deu-se a seguinte: de que forma as tecnologias da informação podem promover educação em saúde na pandemia da coronavírus?

O levantamento se deu através das bases de dados: Base de Dados de Enfermagem (BDENF via BVS), Medical Literature Analysis and Retrieval System Online (MEDLINE via PUBMED) e Literatura Latino-americana e do Caribe em Ciências da Saúde (LILACS via BVS). Para esse trabalho, foram considerados como critérios de inclusão os artigos originais disponíveis por meio eletrônico em português e/ou em inglês e os que estão disponíveis entre o ano de 2020 e 2021, onde correspondem ao tempo de pandemia por covid-19. Como critério de exclusão, foi adotado artigos que não tratam da temática proposta, artigos duplicados, teses, monografias, dissertações, revisão, livros e que não apresenta o texto completo.

A partir disso, o método de busca foi desenvolvido por meio da PICO (Population, intervention, 
comparison, outcomes), no qual Population: coronavírus; Intervention: tecnologias da informação; Comparison: não há e Outcomes: educação em saúde. A seleção dos descritores controlados e não controlados foram através dos descritores em ciências da saúde (DeCS), e no Medical Subject Headings (MeSH) (Tabela 01). Foram cruzados através do operador booleano “AND” para busca simultânea dos assuntos.

Tabela 1. Descritores controlados e não controlados utilizados para busca de dados, Pedreiras, Brasil, 2021.

DECS

\begin{tabular}{|c|c|c|}
\hline \multicolumn{2}{|r|}{ Descritor controlado } & Descritor não controlado \\
\hline $\mathrm{P}$ & Coronavírus & Coronavirus do Coelho \\
\hline I & Tecnologias da informação & $\begin{array}{l}\text { ICT; Tecnologia da Informação e } \\
\text { Comunicação; Tecnologia de Informação; } \\
\text { Tecnologias da Informação; Tecnologias } \\
\text { da Informação e Comunicação; } \\
\text { Tecnologias da Informação e } \\
\text { Comunicações; Tecnologias de } \\
\text { Informação; TIC em Saúde; TIC na Saúde }\end{array}$ \\
\hline $\mathrm{C}$ & - & - \\
\hline $\mathrm{O}$ & Educação em saúde & $\begin{array}{l}\text { Educar para a Saúde; Educação para a } \\
\text { Saúde; Educação para a Saúde } \\
\text { Comunitária; Educação Sanitária }\end{array}$ \\
\hline \multicolumn{3}{|c|}{ MESH } \\
\hline \multicolumn{2}{|r|}{ Descritor controlado } & Descritor não controlado \\
\hline $\mathrm{P}$ & Coronavirus & $\begin{array}{l}\text { Coronaviruses; Coronavirus, Rabbit; } \\
\text { Rabbit Coronavirus; Coronaviruses, } \\
\text { Rabbit; Rabbit Coronaviruses }\end{array}$ \\
\hline I & Information Technology & $\begin{array}{l}\text { Information Technologies; Technology, } \\
\text { Information }\end{array}$ \\
\hline $\mathrm{C}$ & - & - \\
\hline $\mathrm{O}$ & Health Education & $\begin{array}{l}\text { Education, Health; Community Health } \\
\text { Education; Education, Community } \\
\text { Health; Health Education, Community }\end{array}$ \\
\hline
\end{tabular}

Fonte: Autores, 2021. 


\section{RESULTADOS E DISCUSSÃO}

Através do levantamento nas bases de dados, foi quantificado no total 224 artigos. Dentre esses, 08 foram eliminados por não estar em português e/ou inglês, 01 foi eliminado pelo método, 01 por estar disponível na modalidade livro, 01 por estar duplicado e 05 por ausência de texto completo. Restaram então, 208 artigos que foram selecionados para a leitura. Após a leitura, 01 foi eliminado por estar duplicado, 152 por não tratar especificamente da temática, 05 por texto incompleto e 15 pelo método. Mediar
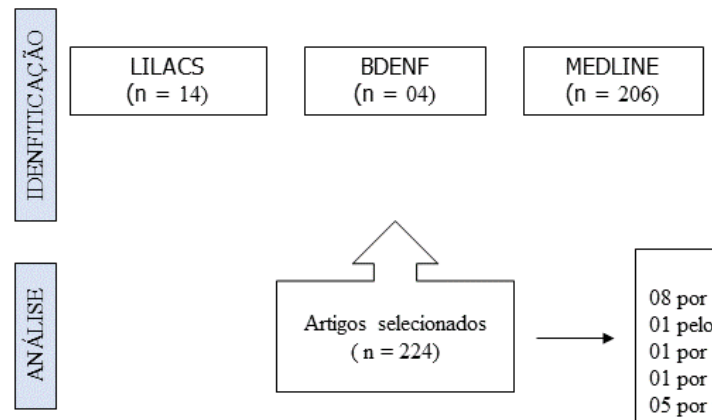

Quadro 1.

$$
\underset{(n=14)}{\text { LILACS }}
$$$$
(n=04)
$$

2021.
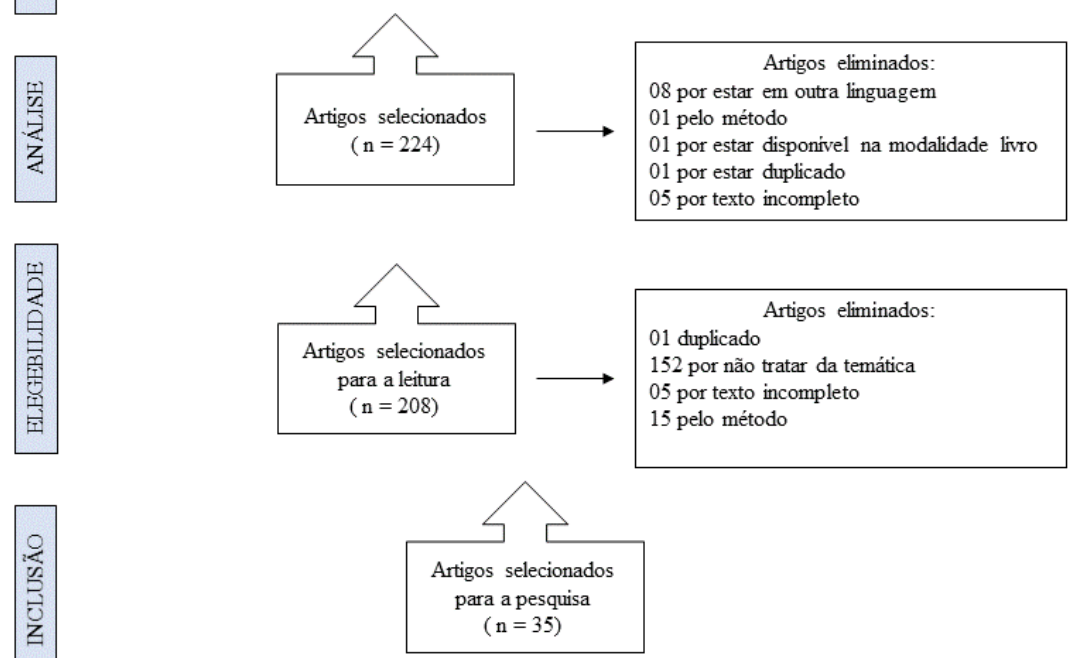

Fonte: Autores, 2021.

Dentre os principais resultados, nota-se de acordo com Soares et al. (2020), que as tecnologias de informação e comunicação contribuem para o repasse das informações e da conversação beneficiando a população, visto que com a atual pandemia a maioria das pessoas estão em suas casas e precisam de conhecimentos que promovam a saúde e auxilie na diminuição da disseminação do vírus.

\section{E - book Tripé do Ensino Superior: Ensino, Pesquisa e Extensão}


Acredita-se também, que o uso das tecnologias para distribuir informações é a forma mais eficaz nesse período, pois proporciona um maior alcance das orientações, podendo chegar à maioria da população. A educação em saúde pode ser realizada de variadas formas, podendo ressaltar a utilização de aplicativos para distribuir conhecimentos sobre ações protetivas, contribuindo para o aumento da segurança no ambiente de trabalho, como por exemplo (SOARES et al., 2020).

Além disso, baseado no estudo de Marasca et al. (2020), as tecnologias contribuíram também para as atividades psicológicas online, no qual foi primordial nesse contexto precário onde muitas pessoas tiveram a saúde mental abalada, possibilitando assim o acesso da população ao cuidado com a mente, visto que com os noticiários sobre a doença, as múltiplas mortes de amigos e familiares e o isolamento social há uma necessidade de ações psicológicas desenvolvida para a sociedade, principalmente para as pessoas mais afetadas psicologicamente pela doença.

Outra atividade tecnológica utilizada atualmente frente a pandemia é o telemonitoramento em saúde, onde é possível acompanhar o paciente e suas condições de saúde sem precisar se locomover e sair de casa, tendo em vista que para a redução da transmissão do coronavírus é primordial o isolamento social. Essa forma de tecnologia é utilizada principalmente com gestantes e idosos que fazem parte do grupo de risco.

Na China, o modo de educação em saúde para distribuir informações importantes sobre a pandemia foi realizado por meio de mensagens de texto, um tipo de tecnologia bastante usada pelas pessoas, onde proporciona a chegada dos conhecimentos sobre prevenção a todo individuo, bem como mensagens de motivações para o enfrentamento desse momento difícil (LUO et al., 2020). Ademais, as lives realizadas pelas redes sociais estão sendo bem vistas na promoção de saúde e prevenção da doença COVID-19 por abranger grande parte da população.

Xie et al (2020), afirma que para o alcance das informações por meio da informática, é fundamental que os educadores e profissionais disseminem esses conhecimentos de maneira criativa para que desperte a atenção da população e assim praticar ações preventivas de forma correta em suas residências. A criatividade é um dos fatores que leva a pessoa a olhar tais informações com outros olhares, visto que é uma forma diferente de disseminar a promoção de saúde, o que faz a sociedade praticar essas ações de promoção.

Para combater a atual pandemia, é preciso que informações corretas sejam disseminadas diariamente, tendo em vista que é um momento onde surge diversas fake News. Com isso, o uso de 
cartilha educativa foi uma maneira acessível para combater essas noticias falsas que podem alterar a qualidade da promoção em saúde, pois através da cartilha ampliou-se as informações seguras, com fontes confiáveis e com embasamento cientifico (SILVA et al., 2020).

De acordo com Valizadeh-Haghi, Khazaal e Rahmatizadeh (2021), as informações em educação em saúde através das tecnologias devem ser divulgadas por meio de conteúdos em linguagem simples que seja de fácil entendimento a toda população para que possa atender suas necessidades, especialmente em tempos de pandemia, pois a população leiga poderá praticar as ações de prevenção e se autocuidar através da divulgação com linguagem simplificada.

As tecnologias de informação podem ser utilizadas também para reduzir estresse e ansiedade no período de pandemia da COVID-19 sendo disseminado estratégias essenciais como a prática de exercício físico, atividades de relaxamento e alimentação saudável para auxiliar na saúde mental dos profissionais da saúde e da população (PINHO et al., 2021).

\section{CONCLUSÃO}

Conclui-se então, que as tecnologias informativas em saúde podem ser utilizadas de diferentes formas para propagar educação em saúde no meio da pandemia da COVID-19. Além disso, é importante ressaltar também que esse meio de transmitir informações em meio as adversidades na atualidade é essencial para que todos tenham acesso a prevenção e cuidados relacionados a doença.

Devido ao fato da COVID-19 ser uma doença de alta transmissão, torna-se irrealizável as ações comunitárias em saúde. Diante do exposto, o uso das tecnologias em tempos de pandemia da COVID19 é urgente e essencial, visto que é uma estratégia inovadora e eficaz para as ações educativas, bem como para a prevenção da doença e para a promoção e recuperação da saúde. Dentre os estudos, vêse a necessidade dessas informações serem de forma criativa para que a população possa se atentar e se prevenir de forma eficaz.

\section{REFERÊNCIAS}

CAVALCANTE, J. et al. COVID-19 no Brasil: evolução da epidemia até a semana epidemiológica 20 de 2020. Epidemiol. Serv. Saúde. v.29, n.4, 2020.

GRANJEIRO, E. et al. Estratégias de ensino à distância para a educação interprofissional em Saúde frente à pandemia COVID-19. REVISA. v.9, p.591-602, 2020.

LUO, Y. et al. Factors influencing health behaviours during the coronavirus disease 2019 outbreak 
in China: an extended information-motivation behaviour skills model. Public Health, v. 185, p. 298-305, 2020.

MANIVA, S. et al. Tecnologias educativas para educação em saúde no acidente vascular cerebral: revisão integrativa. Rev Bras Enferm. v.71(suppl 4):1824-32., 2018

MARASCA, A. R. et al. Avaliação psicológica online: considerações a partir da pandemia do novo coronavírus (COVID-19) para a prática e o ensino no contexto a distância. Estud. psicol. Campinas, v. 37, e200085, 2020.

PIMENTEL, R. M. M. et al. A disseminação da covid-19: um papel expectante e preventivo na saúde global. J Hum Growth Dev. v. 30, n. 1, p. 135-140, 2020.

PINHO, L. et al. O uso de estratégias de promoção da saúde mental por enfermeiros para reduzir a ansiedade, estresse e depressão durante o surto de COVID-19: uma perspectiva. Pesquisa Ambiental, v. 195, e:110828, 2021.

SANTOS, C. M. C.; PIMENTA, C. A. M.; NOBRE, M. R. C. A estratégia pico para a construção da pergunta de pesquisa e busca de evidências. Rev Latino-am Enfermagem, v. 15, n. 3, maiojunho, 2007.

SILVA, R. C. R. et al. Construção de cartilha educativa sobre cuidados com crianças frente à pandemia covid-19: relato de experiência. Rev baiana enferm, v. 34:e37173, 2020.

SOARES, D. C. et al. Tecnologias da informação e comunicação na educação em saúde acerca do Coronavírus: relato de experiência. J. nurs. health., v. 10:e20104027, 2020.

SOUZA, M. T.; SILVA, M. D.; CARVALHO, R. Revisão integrativa: o que fazer. Einstein, v.8, n. 1, p. $102-6,2010$.

VALIZADEH-HAGHI, S; KHAZAAL, Y; RAHMATIZADEH, S. Health websites on COVID-19: are they readable and credible enough to help public self-care? Journal of the Medical Library Association, v. 109, n. 1, January, 2021.

XIE, B. et al. When Going Digital Becomes a Necessity: Ensuring Older Adults' Needs for Information, Services, and Social Inclusion During COVID-19. JOURNAL OF AGING \& SOCIAL POLICY, v. 00, N 00, p. 1-11, 2020. 